\title{
Lessons for Patient Engagement in Research in Low- and Middle-Income Countries
}

\author{
Ana Janic $\cdot$ Kahaki Kimani $\cdot$ Isabel Olembo $\cdot$ Helen Dimaras
}

Received: December 19, 2019 / Published online: March 28, 2020

(C) The Author(s) 2020, corrected publication 2021

\section{ABSTRACT}

Patient engagement in research is marked by partnership between clinicians, scientists, and people with lived experience of a disease, who jointly develop and implement research and disseminate results. Patient engagement in research has been shown to lead to more impactful and relevant findings. There is a global need for quality research contextualized for lowand middle-income countries (LMICs). Patient involvement in research could address this need, yet it remains a practice more commonly

Enhanced digital features To view digital features for this article go to https://doi.org/10.6084/m9.figshare. 11968935.

A. Janic $\cdot$ H. Dimaras $(\bowtie)$

Institute of Medical Sciences, University of Toronto, Toronto, ON, Canada

e-mail: helen.dimaras@sickkids.ca

A. Janic $\cdot$ H. Dimaras

Department of Ophthalmology and Vision Sciences, The Hospital for Sick Children, Toronto, ON, Canada

A. Janic $\cdot$ H. Dimaras

Child Health Evaluative Sciences Program, The Hospital for Sick Children, Toronto, ON, Canada

\section{A. Janic · H. Dimaras}

The Centre for Global Child Health, SickKids

Research Institute, Toronto, ON, Canada employed in high income countries. In this paper, the authors explore LMIC-specific challenges and opportunities for patient engagement in research. Limitations to patient engagement in research include gaps in health infrastructure, socioeconomic status, cultural stigma, and uncertain roles. Potential solutions to address these challenges include strategic national and international research partnerships, initiatives to combat stigma, and sensitization and training of stakeholders in patient engagement in research. Reflecting on their patient engagement experience with eye cancer research in Canada and Kenya, and supported by evidence of patient engagement in other low-resource settings, the authors provide a roadmap for patient engagement in research in LMICs.

\section{K. Kimani}

Department of Ophthalmology, University of Nairobi, Nairobi, Kenya

\section{Olembo}

Syndications and Agency Operations Department, Eastern and Southern African Trade and Development Bank, Nairobi, Kenya

\section{H. Dimaras}

Department of Ophthalmology and Vision Sciences, University of Toronto, Toronto, ON, Canada

\section{H. Dimaras}

Division of Clinical Public Health, University of

Toronto, Toronto, ON, Canada 
Keywords: Global health; Kenya; Low- and middle-income countries; Patient engagement; Patient-partnered research; Retinoblastoma

\section{Key Summary Points}

Patient engagement in research refers to meaningful inclusion of patients as partners in research. When patients are engaged in research, the knowledge produced is more relevant and more likely to be implemented into healthcare practice.

Patient engagement in research is a practice largely limited to high-income countries, yet could have real impacts in solving health challenges in low- and middle-income countries.

We highlight four major challenges to patient engagement in research: gaps in health infrastructure, low socioeconomic status, cultural stigma, and uncertain roles.

Patient mobilization, international collaboration, roles for champions and peer-to-peer support, and education of stakeholders are discussed as solutions to the identified challenges.

\section{INTRODUCTION}

Traditionally, patients (i.e., here defined as individuals with lived experience of disease, including informal caregivers [1]) have been regarded simply as recipients of healthcare services. However, there is a paradigm shift taking place, in which patients are considered to be active participants in shaping their personal health outcomes [2-4]. Patient engagement in healthcare has been shown to strengthen the patient-physician relationship, promote communication about topics that matter to patients, and help patients better understand their care [5], thereby facilitating better care overall [6].
More recently, patient engagement in research has become a precursor to patientcentered care [7]. It promotes active and meaningful involvement of patients as equal partners in research and requires tight collaboration between researchers and patients throughout all phases of research. Roles for patients go beyond the classic role of a research subject and can include leadership or consultation during the decision-making process to choose relevant research questions or study designs; development of study materials (e.g., questionnaires, consent forms); collection of data (e.g., serving as a focus group facilitator); or dissemination of research results (e.g., conference presentations, social media posts) $[1,8]$. Early and frequent engagement of patients on research teams produces studies which are valued by all stakeholders and results in knowledge that is implemented more rapidly across healthcare systems [4].

Patient engagement in research is actively being implemented by the scientific community, as funding bodies create new research guidelines with incentives to include patient partnerships [8]. It is becoming more common for patient representatives to sit on funding panels and have direct input into how funds are allocated [8]. In Canada, the Strategy for Patient-Oriented Research (SPOR) serves to foster patient-researcher partnerships in order to improve research outcomes and direct research funding to studies which are co-created with patients [1]. SPOR follows in the footsteps of similar efforts in other high-income countries (HICs) that promote research involvement of patients, such as the Patient-Centered Outcomes Research Institute in the USA [9] and the INVOLVE in the UK [10].

Patient engagement in research is a growing movement, with demonstrated benefits that could be useful in a global health context, yet it remains primarily practiced in HICs. In this paper, we explore the challenges and opportunities associated with implementing and sustaining patient engagement in research in lowand middle-income countries (LMICs). The authors reflect on their experiences with implementing patient-oriented research in Canada and Kenya surrounding the childhood 
eye cancer retinoblastoma; this reflection is based on previously conducted research and this article does not contain any studies with human participants or animals performed by any of the authors. With supportive evidence from the published literature, the authors propose a framework for sustainable patient engagement in research for LMICs.

\section{CASE STUDY: PATIENT ENGAGEMENT IN RETINOBLASTOMA RESEARCH}

Retinoblastoma is a rare, intraocular cancer that arises in the infant retina. Approximately 8000 children globally are diagnosed with retinoblastoma annually [11]. While curable, retinoblastoma has lifelong consequences that can affect vision and quality of life. Approximately half of survivors have the heritable form of retinoblastoma, conferring risk of second cancers and passing on the risk of cancer to future offspring. In Canada, perhaps because of this lifelong effect of the cancer, patients with retinoblastoma have been strongly engaged in healthcare and research. In 2008, a national strategy involved patients with retinoblastoma in the development of novel clinical care guidelines [12]. In 2016, the Canadian Retinoblastoma Patient Engagement Strategy [13] was created to facilitate more robust patient engagement in retinoblastoma research. The strategy is governed by the Canadian Retinoblastoma Research Advisory Board (CRRAB), which is composed of patients, healthcare professionals, researchers, and patient engagement experts [13]. CRRAB members actively participate in working groups to develop research ideas, scientific protocols, and apply for grants. Patients can also participate as retinoblastoma champions, who promote CRRAB activities and promote research engagement. Ultimately, CRRAB participation and involvement is rewarding, not only to researchers but also for the patients [14].

Despite being a curable cancer, the survival of patients with retinoblastoma in LMICs is low, owing to poor awareness of the early signs of disease among the public, and immature health systems ill-equipped to provide optimal care. Given the relatively high burden of retinoblastoma in Kenya, Canadian experts were invited to collaborate with Kenyan leaders in retinoblastoma to try and improve local survival rates [15]. In 2008, the Kenyan National Retinoblastoma Strategy (KNRbS), a multidisciplinary partnership between Kenya and Canada, was launched with aims to optimize and improve retinoblastoma care in Kenya $[15,16]$. Patients and their families were integral members of the strategy, which also included clinicians, community health workers, and policy makers [17]. By sharing their experiences and views through annual $\mathrm{KNRbS}$ meetings and working groups, patients contributed to the design and development of retinoblastoma awareness campaigns and healthcare delivery centered on retinoblastoma genetics $[18,19]$ and histopathology services [20]. Since the initiation of KNRbS, the age at diagnosis for patients with bilateral retinoblastoma has decreased from 24.4 to 16.8 months of age, and for unilateral patients from 35.9 to 24 months of age, figures more comparable to those observed in HICs and linked to better survival [21].

While patients were significantly engaged in advocacy and clinical care initiatives of the $\mathrm{KNRbS}$, patient engagement in research was limited to non-existent. Several challenges to patient engagement in research were identified, including gaps in health infrastructure, low socioeconomic status, cultural stigma, and uncertain roles. We propose a model to solve these challenges, and foster a culture of patient engagement in research in resource-limited settings (Fig. 1).

\section{CHALLENGES OF PATIENT ENGAGEMENT IN KENYA}

\section{Gaps in Health Infrastructure}

It is well known that several barriers exist to accessing healthcare services in LMICs, such as distance to nearest health facility, lack of resources, and complex bureaucratic referral systems [22]. These barriers are linked to poor 


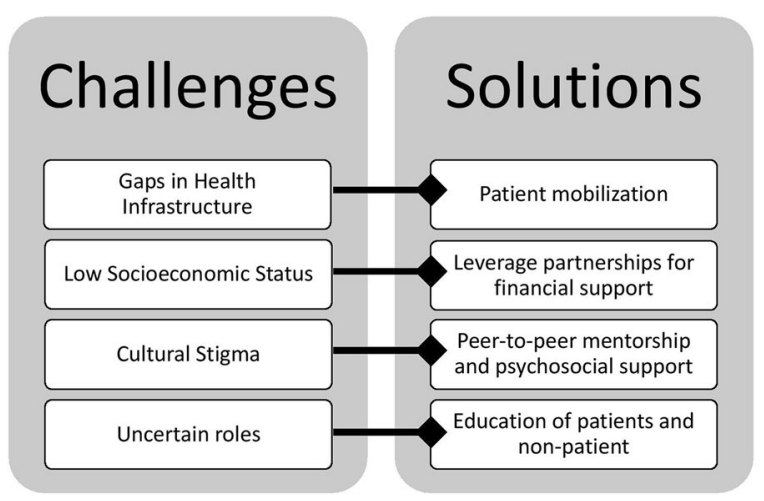

Fig. 1 Framework for patient engagement in research in low- and middle-income countries. Four challenges were identified which impede patient engagement in research: gaps in health infrastructure, low socioeconomic status, cultural stigma, and uncertain roles. Solutions are offered for addressing each of these challenges

health outcomes [22-24]. Therefore, if access to healthcare services for basic treatment is limited, and health outcomes remain poor as a result, the common notion is that it is unlikely and even unwise to focus efforts towards less "urgent" initiatives, like patient involvement in research.

There are several instances where even when health outcomes of citizens are poor, effective engagement is achieved and even warranted. For example, the Kenyan government engaged community members for the development of their cancer health policy, even with gaps in health infrastructure persisting [2]. This led us to ask: why is this not the case then for patient engagement in research? We argue, if curative treatment is currently unavailable, then research should focus on making families more comfortable in their current state. A way to do this is to listen to patients' personal experiences and incorporate their ideas into research, which has been shown to positively influence quality of life $[4,14]$.

For patient engagement in research to grow, it needs to align with a system that welcomes patient and caregiver involvement and makes participation accessible and easy. In many cases systems in low-resource settings can improve with patient mobilization to advocate for change. Patients with breast cancer have started doing so by expressing their desire for active inclusion in care and for the opportunity to voice their experiences [25]. The KNRbS was one type of forum or "space" where direct engagement and collaboration among patients with retinoblastoma, healthcare workers, and academics was possible, and revealed that patients were interested in participating in research [15]. Qualitative methods can be used to inform the underpinning of a patient engagement initiative, particularly when it comes to identifying local factors that motivate patients to engage in research [26]. In Canada, conducting focus groups with patients with retinoblastoma revealed important insight into their current views, understanding, and apprehensions regarding engaging in research [27] and mobilized the development of a framework for sustainable patient engagement [28]. It is in this space, where there is balance between optimism that a patients' voice will change a situation and a system that is open to the patient, that patient engagement can grow.

\section{Low Socioeconomic Status}

If an individual's financial situation can be a determinant of their health-seeking behavior, then it follows that it can also affect their engagement in research $[23,29,30]$. Patients often incur out-of-pocket medical costs for diagnosis, travel to healthcare facilities, and treatment [31], which places a high burden on patients of low socioeconomic status. Since engagement in research also carries financial costs, as it involves participants taking time off work, attending conferences, and traveling to meetings [15], individuals of low socioeconomic status face increased strain. Furthermore, in some situations, researchers may hesitate to reach out to patients for potential involvement, assuming engagement is prohibitive because of the financial stress it imposes. All these factors, in turn, could lead to limited patient engagement in research.

In order to help alleviate financial stress on the patient and increase the opportunity for engagement in research, researchers offer compensation for patients through research grants or other project funding. The rationale for this 
is that investing in patient engagement in research can lead to reduction in overall healthcare "waste" by supporting research that is valuable to patients and minimizing research and treatment costs that lead to poor use of resources $[8,14]$.

However, given the limited funding climate in LMICs for research, it is unrealistic at this time to expect organizations in resource-constrained settings to have the capacity to absorb these patient engagement costs. Potentially such costs could be covered through partnerships with international bodies with more resources for patient engagement, a solution frequently used in LMIC settings [3]. For example, through partnerships with Canadian institutions, KNRbS was able to cover the costs of patient travel to and participation in annual meetings [15]. Similarly, the community engagement to inform cancer health policy in Kenya was funded via an international partnership with the UK, and nationally, with local Kenyan non-profit and private organizations [2].

\section{Cultural Stigma}

In many settings, the diagnosis of an illness can lead to unpleasant effects on community life, such as social isolation and stigmatization $[24,25,32]$. Patients with cancer report experiencing social stigma, with consequences such as termination of employment, familial shame, and social rejection [23, 31, 33]. In some regions, beliefs persist that cancer is caused by witchcraft and, therefore, patients prefer to keep their disease private [19]. Additionally, some patients experience feelings of defeat and fatalistic attitudes when describing their experiences with cancer, many of whom ignored early signs of their cancer and/or delayed care as a result of misconceptions of cancer as incurable [32]. For patients with retinoblastoma who have had an enucleation (eye removal) to save their life, living with a prosthetic eye, or an empty socket when a prosthetic is not available, poses additional challenges to societal integration. These social consequences may deter patient involvement in research, as patients may not want to identify themselves with a particular health concern or illness.

Despite this challenge, we need to look no further than the participatory research conducted in the field of HIV to see successful patient research engagement in LMICs. For many years now, researchers have been able to successfully combat stigma surrounding patients living with HIV in low-resource settings [34]. By creating HIV-positive patient champions that conduct research interviews, researchers have removed barriers by including people with lived experience as members of research teams. Reduction of stigma and increased engagement have also been achieved by providing confidential settings for HIV-positive individuals to engage in projects with support from HIV-positive patient champions and mental health workers [35].

Education and psychosocial support are additional aspects of combatting social stigma of disease. In the KNRbS, healthcare workers were trained as Child Life specialists to provide families with child-centered psychosocial support [15]. Child Life specialists provide support and education, to children and their families, to help children address stigma and cope with difficult medical procedures. Some of this work addressed access to and care of artificial eyes, training young patients to be their own champions and normalize their "special eye" [14]. Thus, with thoughtful and concerted effort, social stigma can start to be erased, and allow for meaningful patient engagement in research.

\section{Uncertain Roles}

Patient engagement in research is a relatively new concept; therefore, the idea of patients as research collaborators may be difficult for patients to understand. Individuals may be more familiar with roles in advocacy or support group leadership for patients. These roles normally do not intersect with research, but rather allow patients to share their experiences, raise public awareness, or fundraise [36].

Patients are often not aware of how they can be effective research collaborators and that their participation in research can be beneficial. They 
often presume their knowledge and training in research is inadequate [37], and therefore not useful. However, patients can be coached to recognize that knowledge and expertise come in many forms, and that lived experience is just as valuable as clinical or research knowledge, even if it is different [8, 37]. In Canada, CRRAB working groups have focused on providing a space for patients to share their lived experience while also connecting them to training and tools to be effective partners, such as online introductory courses on research [1, 14].

In addition, the idea of patients as research collaborators may be difficult for non-patients (i.e., physicians, researchers, allied healthcare professionals) to accept. For example, it may be challenging for patients to share their experience if non-patient attitudes to patient participation are dismissive [37]. Patients have expressed the need for a culture shift to reweigh the scales of power, and encourage active listening on the part of health and research professionals such that patient values are incorporated into treatment plans or research $[8,38,39]$. Patients suggest that positive clinician attitudes and behaviors towards them are critical for their overall engagement [39].

Training, for both researchers and patients, on the ins and outs of patient engagement in research, could help clarify roles and lead to more effective engagement. Additionally, it could prevent ethical concerns with the practice, such as tokenistic involvement of patients in research (i.e., patient inclusion purely motivated by pursuing research funding, without commitment to true research partnership $[3,40])$. As shown through the use of participatory research methods, the very process of patient engagement in research can promote equity in the research process, such that patient voices are heard and research outcomes remain relevant to the intended beneficiaries [41, 42]. Additionally, in some LMIC contexts, where research ethics infrastructure is less robustly developed than in HICs, patient partnership to develop research ethics policies and tools (e.g., informed consent protocols) could have a significant impact in promoting equity and protecting vulnerable groups $[41,42]$.
A multidisciplinary and multisectoral team, which cultivates cross talk on a leveled platform, could work to establish a collaborative space between patients and non-patients and help clarify roles. In designing a study on cervical cancer in Kenya, researchers obtained community support and trust by holding stakeholder meetings that served as an open forum to discuss study objectives and overall implementation of screening [24]. Overall, establishing well-defined roles for patient participation and providing a space for collaboration is critical to facilitating patient engagement in research.

\section{CONCLUSION}

This paper has considered the importance of patient engagement in LMICs and has identified four challenges impeding implementation: gaps in health infrastructure, low socioeconomic status, cultural stigma, and uncertain roles. For each of these challenges, potential solutions have been put forth. First, the perception that gaps in healthcare infrastructure preclude patient engagement can be confronted by first mobilizing patients through simple and tangible ways in supportive environments. Second, to address the challenge of socioeconomic status, research groups in LMICs can seek collaborations with international partners where patient engagement in research is gaining traction and supported financially. Third, to mitigate social isolation and stigma of patients, champions can serve as a positive, public face to illness, and peer-to-peer mentors could provide individualized psychosocial support. Finally, uncertain roles of patients in research can be tackled by educating patient and non-patient stakeholders on the benefits of patient engagement in research, and providing a forum for establishing shared goals as well as well-defined roles and responsibilities to achieve them.

Empowerment and inclusion of patients as partners in research is not a luxury of the western world, but an imperative to improve health even in LMICs. By looking to and mirroring successful patient engagement initiatives, locally and globally, research groups in 
resource-constrained settings can start small and build forward. Even in LMICs, ample opportunity exists for patients to help set research priorities, champion research initiatives, take a leading role in data collection, and help disseminate results. With some targeted support, patients can be empowered to such roles and help change the research landscape globally.

\section{ACKNOWLEDGEMENTS}

A patient co-author is included on this manuscript.

Funding. Ana Janic was funded by the Queen Elizabeth II Diamond Jubilee Foundation. No additional funding was received for the publication of this article.

Authorship. All named authors meet the International Committee of Medical Journal Editors (ICMJE) criteria for authorship for this article, take responsibility for the integrity of the work as a whole, and have given their approval for this version to be published.

Author Contributions. Conception: Helen Dimaras. Acquisition of data: Ana Janic, Helen Dimaras, Kahaki Kimani, Isabel Olembo. Drafting of manuscript: Ana Janic. Critical revision of manuscript: Ana Janic, Helen Dimaras, Kahaki Kimani, Isabel Olembo. All authors read and approved the final manuscript.

Disclosures. Ana Janic, Kahaki Kimani, Isabel Olembo, and Helen Dimaras all declare that they have no conflict of interest.

Compliance with Ethics Guidelines. This article is based on previously conducted studies and does not contain any studies with human participants or animals performed by any of the authors.

Peer Review. Please note, contrary to the journal's standard single-blind peer review process, as a commentary this article underwent review by a member of the journal's Editorial Board.

Data Availability. Data sharing is not applicable to this article as no datasets were generated or analyzed during the current study.

Open Access. This article is licensed under a Creative Commons Attribution-NonCommercial 4.0 International License, which permits any non-commercial use, sharing, adaptation, distribution and reproduction in any medium or format, as long as you give appropriate credit to the original author(s) and the source, provide a link to the Creative Commons licence, and indicate if changes were made. The images or other third party material in this article are included in the article's Creative Commons licence, unless indicated otherwise in a credit line to the material. If material is not included in the article's Creative Commons licence and your intended use is not permitted by statutory regulation or exceeds the permitted use, you will need to obtain permission directly from the copyright holder. To view a copy of this licence, visit http://creativecommons.org/licenses/by$\mathrm{nc} / 4.0 /$.

\section{REFERENCES}

1. Canadian Institutes of Health Research. Strategy for patient-oriented research. http://www.cihr-irsc.gc. ca/e/45851.html. Accessed 16 Sep 2019.

2. O'Meara WP, Tsofa B, Molyneux S, et al. Community and facility-level engagement in planning and budgeting for the government health sector-a district perspective from Kenya. Health Policy. 2011;99:234-43. https://doi.org/10.1016/j. healthpol.2010.08.027.

3. Cook N, Siddiqi N, Twiddy M, et al. Patient and public involvement in health research in low and middle-income countries: a systematic review. BMJ Open. 2019;9:e026514. https://doi.org/10.1136/ bmjopen-2018-026514.

4. Hibbard JH, Greene J. What the evidence shows about patient activation: better health outcomes and care experiences; fewer data on costs. Health Aff (Millwood). 2013;32:207-14. https://doi.org/10. 1377/hlthaff.2012.1061. 
5. Epstein RM, Street RL. The values and value of patient-centered care. Ann Fam Med. 2011;9:100-3. https://doi.org/10.1370/afm.1239.

6. Boivin A, Richards T, Forsythe L, et al. Evaluating patient and public involvement in research. BMJ. 2018. https://doi.org/10.1136/bmj.k5147.

7. Stollenwerk D, Kennedy LB, Hughes LS, et al. A systematic approach to understanding and implementing patient-centered care. Fam Med. 2019;51: 173-8. https://doi.org/10.22454/FamMed.2019. 320829.

8. Dean S, Mathers JM, Calvert M, et al. "The patient is speaking": discovering the patient voice in ophthalmology. $\mathrm{Br} \mathrm{J}$ Ophthalmol. 2017;101:700-8. https://doi.org/10.1136/bjophthalmol-2016309955.

9. Patient-Centered Outcomes Research Institute. Patient engagement. https://www.pcori.org/aboutus/our-programs/engagement. Accessed 27 Aug 2019.

10. INVOLVE. National Institute for Health Research. https://www.invo.org.uk. Accessed 17 Sep 2019.

11. Dimaras H, Corson TW, Cobrinik D, et al. Retinoblastoma. Nat Rev Dis Primer. 2015;1:15021. https://doi.org/10.1038/nrdp.2015.21.

12. National Retinoblastoma Strategy. Canadian guidelines for care. Can J Ophthalmol. 2009;44: S9-47. https://doi.org/10.3129/i09-194.

13. Canadian Retinoblastoma Research Advisory Board. http://www.rbcanadaresearch.com/. Accessed 2 Sept 2019.

14. White E, Baddeliyanage R, Shaikh F, et al. Meaningful patient engagement in research: lessons from retinoblastoma. Pediatrics. 2019;143:e20182166. https://doi.org/10.1542/peds.2018-2166.

15. Daisy's Eye Cancer Fund and The Kenyan National Retinoblastoma Strategy Group, Hill JA, Kimani K, et al. Achieving optimal cancer outcomes in East Africa through multidisciplinary partnership: a case study of the Kenyan National Retinoblastoma Strategy group. Glob Health. 2016;12:454. https:// doi.org/10.1186/s12992-016-0160-1.

16. Reddy A, Kimani K. A national retinoblastoma network: experiences in Kenya and the UK. Community Eye Health. 2018;31:5-6.

17. Department of Ophthalmology, University of Nairobi. Retinoblastoma best practice guidelines. http://guidelines.health.go.ke:8000/media/BestPractice-Retinoblastoma_September-2014.pdf. Accessed 27 Sept 2019.
18. He LQ, Njambi L, Nyamori JM, et al. Developing clinical cancer genetics services in resource-limited countries: the case of retinoblastoma in Kenya. Public Health Genomics. 2014;17:221-7. https:// doi.org/10.1159/000363645.

19. Lee S, Gedleh A, Hill JA, et al. In their own words: a qualitative study of Kenyan Breast Cancer Survivors' knowledge, experiences, and attitudes regarding breast cancer genetics. J Glob Oncol. 2018. https://doi.org/10.1200/jgo.17.00061.

20. Qaiser S, Limo A, Gichana J, et al. Design and implementation of the retinoblastoma collaborative laboratory. Ocul Oncol Pathol. 2017;3:73-82. https://doi.org/10.1159/000451000.

21. Namweyi Nandasaba R, Kimani K, Njambi L. Outcome of Globe Preservation Therapy in Patients with Bilateral Retinoblastoma at the Kenyatta National Hospital, Kenya. 2015. http://erepository. uonbi.ac.ke/handle/11295/80851. Accessed 15 Sept 2019.

22. Makau-Barasa LK, Greene SB, Othieno-Abinya NA, et al. Improving access to cancer testing and treatment in Kenya. J Glob Oncol. 2018. https://doi.org/ 10.1200/jgo.2017.010124.

23. Njuguna F, Martijn H, Langat S, et al. Factors influencing time to diagnosis and treatment among pediatric oncology patients in Kenya. Pediatr Hematol Oncol. 2016;33:186-99. https://doi.org/ 10.3109/08880018.2016.1169566.

24. Buchanan Lunsford N, Ragan K, Lee Smith J, et al. Environmental and psychosocial barriers to and benefits of cervical cancer screening in Kenya. Oncologist. 2017;22:173-81. https://doi.org/10. 1634/theoncologist.2016-0213.

25. Sayed S, Ngugi AK, Mahoney MR, et al. Breast cancer knowledge, perceptions and practices in a rural community in Coastal Kenya. BMC Public Health. 2019. https://doi.org/10.1186/s12889-019-6464-3.

26. Phoenix M, Nguyen T, Gentles SJ, et al. Using qualitative research perspectives to inform patient engagement in research. Res Involv Engagem. 2018;4:20. https://doi.org/10.1186/s40900-0180107-1.

27. Moses C, Flegg K, Dimaras H. Patient knowledge, experiences and preferences regarding retinoblastoma and research: a qualitative study. Health Expect. 2020. https://doi.org/10.1111/hex.13043.

28. Gelkopf MJ, Avramov I, Baddeliyanage R, et al. The Canadian retinoblastoma research advisory board: a framework for patient engagement. Res Involv Engagem. 2020;6:7. https://doi.org/10.1186/ s40900-020-0177-8. 
29. Atieno OM, Opanga S, Martin A, et al. Pilot study assessing the direct medical cost of treating patients with cancer in Kenya; findings and implications for the future. J Med Econ. 2018;21:878-87. https:// doi.org/10.1080/13696998.2018.1484372.

30. Ellen M, Shach R, Kok M, et al. There is much to learn when you listen: exploring citizen engagement in high- and low-income countries. World Health Popul. 2017;17:31-42. https://doi.org/10. 12927/whp.2017.25307.

31. Lim JNW, Ojo AA. Barriers to utilisation of cervical cancer screening in Sub Sahara Africa: a systematic review. Eur J Cancer Care (Engl). 2017;26:e12444. https://doi.org/10.1111/ecc.12444.

32. De Ver Dye T, Bogale S, Hobden C, et al. A mixedmethod assessment of beliefs and practice around breast cancer in Ethiopia: implications for public health programming and cancer control. Glob Public Health. 2011;6:719-31. https://doi.org/10. 1080/17441692.2010.510479.

33. Rosser JI, Njoroge B, Huchko MJ. Cervical cancer stigma in rural Kenya: what does HIV have to do with it? J Cancer Educ. 2016;31:413-8. https://doi. org/10.1007/s13187-015-0843-y.

34. Lorway R, Thompson LH, Lazarus L, et al. Going beyond the clinic: confronting stigma and discrimination among men who have sex with men in Mysore through community-based participatory research. Crit Public Health. 2014;24:73-87. https://doi.org/10.1080/09581596.2013.791386.

35. Hosek SG, Harper GW, Lemos D, et al. Project ACCEPT: evaluation of a group-based intervention to improve engagement in care for youth newly diagnosed with HIV. AIDS Behav. 2018;22:2650-61. https://doi.org/10.1007/s10461-018-2034-4.

36. $\mathrm{Hu} \mathrm{A}$. Reflections: the value of patient support groups. Otolaryngol Neck Surg. 2017;156:587-8. https://doi.org/10.1177/0194599817697030.

37. Carroll S, Embuldeniya G, Abelson J, et al. Questioning patient engagement: research scientists'; perceptions of the challenges of patient engagement in a cardiovascular research network. Patient Prefer Adherence. 2017;11:1573-83. https://doi. org/10.2147/PPA.S135457.

38. Ngugi CW, Boga H, Muigai AWT, et al. Factors affecting uptake of cervical cancer early detection measures among women in Thika, Kenya. Health Care Women Int. 2012;33:595-613. https://doi.org/ 10.1080/07399332.2011.646367.

39. Wachira J, Genberg B, Kafu C, et al. Experiences and expectations of patients living with HIV on their engagement with care in Western Kenya. Patient Prefer Adherence. 2018;12:1393-400. https://doi. org/10.2147/PPA.S168664.

40. Lemonsky F. Service user involvement in research. Lancet Psychiatry. 2015;2:780. https://doi.org/10. 1016/S2215-0366(15)00365-X.

41. Justo L. Participatory research: a way to reduce vulnerability. Am J Bioeth. 2004;4:67-8. https:// doi.org/10.1080/15265160490497119.

42. Liabo K, Ingold A, Roberts H. Co-production with "vulnerable" groups: balancing protection and participation. Health Sci Rep. 2018;1:e19. https://doi. org/10.1002/hsr2.19. 